\begin{tabular}{|c|}
\hline Ra Ximhai \\
Revista de Sociedad, Cultura y Desarrollo \\
Sustentable
\end{tabular}

Ra Ximhai

Universidad Autónoma Indígena de México

ISSN: 1665-0441

México

2013

HUERTO ESCOLAR: ESTRATEGIA EDUCATIVA PARA LA VIDA

Benito Rodríguez-Haros; Enriqueta-Tello-García y Salvador-Aguilar-Californias

Ra Ximhai, enero - abril, año/Vol. 9, Número 1

Universidad Autónoma Indígena de México

Mochicahui, El Fuerte, Sinaloa. pp. 25-32.

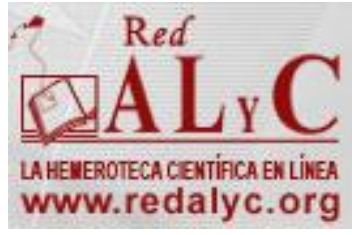




\title{
HUERTO ESCOLAR: ESTRATEGIA EDUCATIVA PARA LA VIDA
}

\section{SCHOLAR GARDEN: EDUCATIONAL STRATEGY FOR LIFE}

\author{
Benito Rodríguez-Haros ${ }^{1}$; Enriqueta-Tello-García ${ }^{2}$ y Salvador-Aguilar-Californias ${ }^{3}$ \\ ${ }^{1}$ Profesor de la Universidad de Guanajuato Campus Celaya-Salvatierra. Privada de Arteaga S/N Salvatierra Guanajuato, CP $38900 .{ }^{2}$ Investigadora \\ Titular del Programa de Estudios para el Desarrollo Rural, Colegio de Posgraduados. Carretera Federal México -Texcoco Km 36.5 , CP 56230. ${ }^{3}$ \\ Director General del Colegio Ateneo, Educación Prescolar. Calle 21 de Marzo Col. San Felipe Tezoyuca, Estado de México. CP 56000.
}

\section{RESUMEN}

Hace aproximadamente cinco años, y preocupados por la erosión del conocimiento vinculado a los procesos productivos de los alimentos, el acceso a ellos y la calidad nutrimental e inocuidad, se estableció el huerto agroecológico "un pasito en grande", donde el uso de los agroquímicos (fertilizantes, insecticidas, herbicidas, etc.) están prohibidos. Todo ello con la colaboración y participación de niños, niñas, padres y madres de familia y personal docente del Colegio Ateneo prescolar de Tezoyuca, Estado de México. La participación de los infantes ha permitido la difusión de la experiencia, y poco a poco la estrategia se ha ampliado a otros espacios educativos.

El huerto escolar se ha convertido en un espacio para fomentar una conciencia ecológica y ambiental que se fortalece a partir de las actividades cotidianas y acciones específicas implementadas. El huerto escolar se fundamenta en una serie de principios filosóficos que ayuda a la reflexión de nuestro aprender-hacer; en términos metodológicos su implementación se sustenta en la ética y principios de la permacultura.

Palabras clave: educación ambiental, agroecología, cultivos orgánicos, cultura ambiental.

\section{SUMMARY}

About five years ago, and worried about the erosion of knowledge related to the process of food production, access and safety, an agroenvironmental vegetable garden was established and named "Un pasito en grande" (A large baby step), where the use of agrochemicals (fertilizers, insecticides, herbicides, etc.) are forbidden. Everything takes place with the participation of boys, girls, fathers and mothers of the Colegio Ateneo nursery school of Tezoyuca, State of Mexico. Childrens' participation has helped spread the word about the experience and little by little, the strategy has spread to other educational spaces.

The school garden has become a space to raise ecological and environmental awareness that is strengthened with daily activities and specific activities that are implemented. The school garden is based on a series of philosophical principles that help reflect upon our learning-doing; in methodological terms, its implementation is based on ethics and on the principles of permaculture.

Key words: ambiental education, agroecology, organic crops, ambiental culture.

\section{INTRODUCCIÓN}

El modelo hegemónico de educación busca formar ciudadanos capaces de producir siguiendo un mismo patrón de comportamiento, repitiendo y reproduciendo recetas tecnológicas de producción, que desempeñen su trabajo o profesión sin ser creativos e innovadores y que estén al servicio del mercado. Romper con este modelo hegemónico, y enfrentar los desafíos de una educación acordes al siglo XXI, requiere formar personas con espíritu crítico, innovador, creativo, con capacidad de analizar, sintetizar y de inferir, así como adaptarse a la velocidad de los cambios de este mundo globalizador. Vivimos en una época de transición y contradicción, en la cual el desenlace contiene altos niveles de incertidumbre y complejidad. Los actuales modelos de producción, transforman el modo y las relaciones de producción, los patrones socioculturales y nuestra vida cotidiana, demandándose una educación que forme hombres y mujeres con las habilidades que la sociedad moderna requiere; sin embargo, no podemos continuar con el mismo patrón de producción y educación, pues se ha puesto en riesgo no solo la vida de la humanidad, sino de toda la vida que habita en el planeta. Tenemos el gran desafío de reconstruirlo y conservar el equilibrio ecológico en el sistema tierra y lograr una salud individual, social y ambiental; la vida humana se desenvuelve en la esfera biológica, de donde los seres humanos satisfacemos nuestras necesidades primarias.

En el sentido filosófico, y desde la educación, se requiere de instrumentos pedagógicos metodológicos complementarios para avanzar en el proceso de la educación y su vinculación sociológica. Cada 
institución, cada sociedad, cada grupo, cada alumno y alumna, cada docente, tendrá la responsabilidad de asumir la filosofía de un modelo que se adapte a sus necesidades de enseñanza-aprendizaje, comprometerse con uno u otro modelo debe implicar un cuestionamiento, un análisis y a la vez se deben proponer acciones que superen las propuestas de los mismos modelos y enganchando teorías postmodernistas, proactivas que coadyuven a la mejora de los procesos de enseñanza. En este contexto, los métodos, los principios pedagógicos, y los enfoques curriculares tendrán otro sentido, al igual que las políticas educativas; la visión de los planes y programas a nivel nacional, comparados con los internacionales, deberán ejercer una presión para reflexionar y actuar en pro de la educación y sus actores en un escenario educativo (Alcorta, 2000).

Coincidimos con Gadotti (2002) cuando plantea que se requiere de una conciencia ecológica y ambiental de toda la humanidad en los procesos educativos, y para que sea realmente efectiva y transformadora, debe ser aplicada a las actividades cotidianas a partir de acciones concretas. Necesitamos de un nuevo paradigma que tenga como fundamento la Tierra, el cual deberá basarse en nuevos valores, nuevas actitudes, un nuevo mirar y actuar.

En la búsqueda de este paradigma y desde la perspectiva de la educación, habrá que ver a la Tierra como un organismo vivo y en evolución, con la finalidad de contribuir a mejorarla desde nuestra casa y desde los espacios en donde los seres humanos interactuamos de manera cotidiana, con sensibilidad y competencia (Gadotti, 2002).

Para nosotros, uno de estos espacios ha sido la implementación del huerto escolar, en donde intentamos poner en práctica un aprendizaje en la acción. Estamos convencidos que el huerto, como estrategia educativa, "es un espacio de comunicación que trasciende, de los aspectos formales de la comunicación oral y escrita, a un intercambio natural-cultural que vincula espacio y tiempo en las relaciones entre la humanidad y la naturaleza" (Tello et al., 20011:51); además, nos induce a implementar pequeños cambios en nuestras vidas, pero haciéndolo de una manera consciente y constante, para lograr transformar no solo nuestra mente, sino la salud física, espiritual y emocional, que se ve reflejado en el bienestar y por supuesto en el entorno donde vivimos; a la vez que se generan una serie de valores sobre la vida, la salud, el trabajo, la participación, la responsabilidad, la autorrealización y la concertación.

Con la intensión de mostrar cómo hemos ido construyendo el huerto, como estrategia educativa, en el presente trabajo nos planteamos los siguientes objetivos: esbozar algunos de los planteamientos que se proponen para abrir nuevos caminos en el ámbito educativo, a partir de la ecopedagogía o pedagogía de la tierra; enunciar los principios éticos y de diseño que tratamos de incorporar en el huerto; y por último, describir los elementos fundamentales que nos han dado la posibilidad de que el huerto pueda convertirse en un espacio estratégico para impulsar una educación integral.

El documento está estructurado en tres apartados, en el primero se describen algunos elementos planteados desde la ecopedagogía o pedagogía de la tierra, que como teoría de la educación promueve el aprendizaje del sentido de las cosas; en el segundo apartado se enuncian los principios éticos y de diseño que se pretenden impulsar en el huerto escolar; y finalmente se enuncian las actividades implementadas en este proceso del aprendizaje no solo en el huerto, sino en el espacio educativo.

\section{Pedagogía de la tierra: necesidad para un mejor estilo de vida}

Desde la pedagogía de la tierra se considera que hemos perdido la posibilidad de ver a la Tierra como el espacio en donde habitamos y que nos da sustento; nos han educado para ver la Tierra como un sistema mecánico, al cual podemos dominar a través del uso de la tecnología de alto consumo energético, haciendo insostenible el modelo de desarrollo, dejando a un lado un mundo real por otro mundo virtual. Por lo cual, es necesario un cambio de paradigma para reeducar y educar de manera diferente. 
La Ecopedagogia constituye una pedagogía para la promoción del aprendizaje del sentido de las cosas, a partir de la vida cotidiana. Se plantea como un nuevo paradigma, que se centra en la vida; es la educación para una actuación responsable hacia y por el ambiente, vinculada al espacio y al tiempo, donde las relaciones entre el ser humano y el ambiente tienen lugar. Implica incorporar los valores y principios que defiende la Carta de la Tierra ${ }^{1}$, y por tanto una redefinición de la curricula (Antunez y Gadotti, 2000).

La pedagogía de la tierra, o ecopedagoía, es un proyecto alternativo global, en donde su preocupación no es la preservación de la naturaleza en sí, o al impacto de las sociedades humanas sobre los ambientes naturales, más bien, es un nuevo modelo de civilización sustentable que implica un cambio en las estructuras económicas, sociales, culturales y espirituales. Se busca el equilibrio del ser humano con él mismo, con el planeta y el universo. Puede verse como un proyecto utópico que pretende cambiar las relaciones humanas, sociales y ambientales de hoy en día. Pero, una utopía como algo inédito y viable, que puede trascender la realidad y buscar la manera de mejorar, y potenciar los saberes y conocimientos necesarios en la construcción de alternativas autónomas (Antunez y Gadotti, 2000; y Gutiérrez y Cruz, 2004).

La ecopedagogia o Pedagogia de la Tierra, parte de un paradigma filosófico que integra el pensamiento de diferentes autores y ofrece un conjunto de conocimientos y valores interdependientes, tales como: educar para pensar en forma global; educar los sentimientos; enseñar sobre la identidad de la Tierra como algo esencial para la condición humana; moldear la conciencia planetaria; educar para el entendimiento y educar para la simplicidad, el cuidado y la paz. Busca las mejores condiciones de vida para tod@s, por medio de la interacción entre la educación para el entorno, el desarrollo económico y el progreso social (Figura 1), para conseguir objetivos naturales, sociales y económicos (Mallart, 2010).

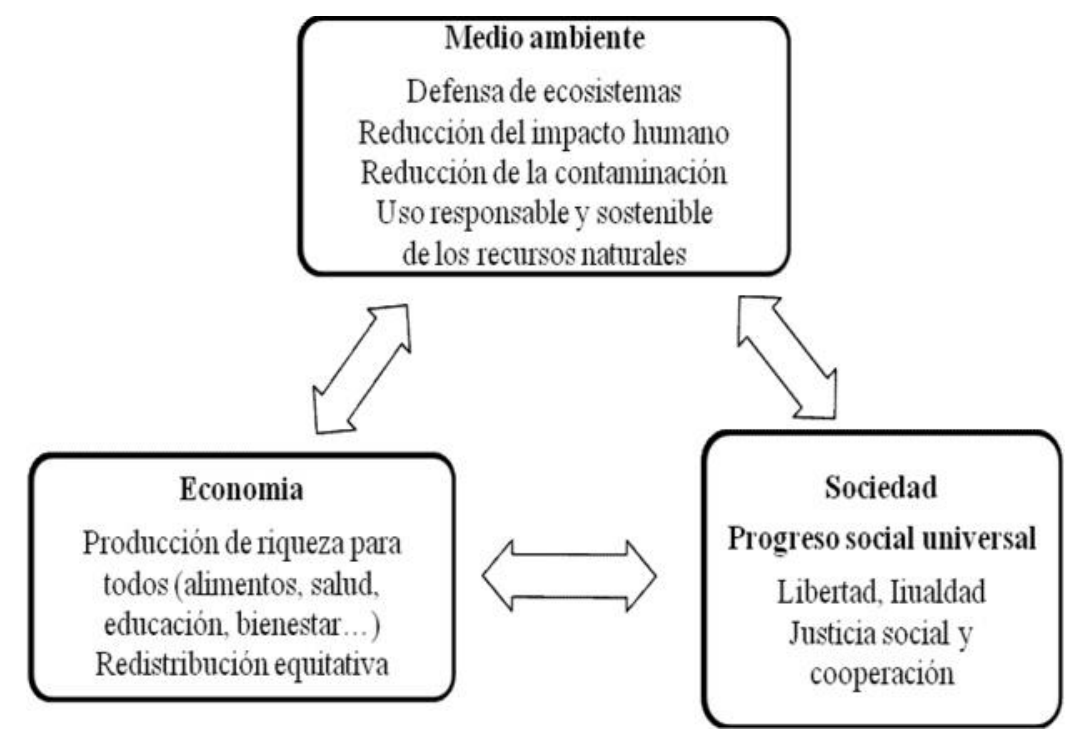

Figura 1. Interacciones a partir de la Ecopedagogía

De acuerdo con Gadotti (2000:32-38), las categorías que ayudan a abrir nuevos caminos en términos educativos, son planetariedad, la sustentabilidad, la virtualidad, la globalización y la transdisciplinariedad. La planetariedad debe llevarnos a sentir y vivir nuestra cotidianidad en conexión con el universo y en relación armónica con nosotros mismos, con los demás seres del planeta y con la naturaleza, considerando sus elementos y su dinámica. La sustentabilidad debe ser un principio interdisciplinario reorientador de la

\footnotetext{
${ }^{1}$ Es una guía ética para el desarrollo sostenible. Se reconoce en el 2003, en la 32a Conferencia General de la UNESCO. Sus principios se pueden resumir de la siguiente manera: 1) Respeto y cuidado por la comunidad de vida; 2) Integridad ecológica; 3) Justicia social y económica y 4) Democracia, no violencia y paz.
} 
educación, del planteamiento escolar, de los sistemas de enseñanza y de los proyectos ecopedagógicos de las escuelas. La virtualidad implica la discusión sobre la educación a distancia y el uso de las computadoras en las escuelas. La globalización se debe reflexionar sobre el proceso de globalización de la economía, la cultura y de las comunicaciones. La transdisciplinariedad engloba y trasciende las disciplinas, sin anularlas, manteniendo la complejidad de lo real.

Sin embargo, estas categorías serían insuficientes para entender la ecopedagogía como teoría de la educación; el mismo autor señala la inclusión de otras categorías ligadas a las esferas de la subjetividad, de la cotidianidad y del mundo vivido, pues estructuran la vida de todos los días, y consideran las prácticas individuales y colectivas, así como las experiencias personales. Dichas categorías no pueden ser exploradas de manera aislada, pues no representarían una revolución científica; de lo contrario si se asocian a otras categorías y otros conceptos, estarían en condiciones de indicarnos un nuevo paradigma. Agrega que en el siglo XXI, las nuevas tecnologías y la ecología no solo representan un cambio de visión del planeta, sino un cambio acerca del futuro común de la humanidad.

Por otro lado, Zingaretti (2008) hace una serie de señalamientos sobre la Ecopedagogia, los cuales se sintetizan en el cuadro 1.

\section{Cuadro1. Planteamientos sobre la ecopedagogía}

\begin{tabular}{ll}
\hline \multicolumn{1}{c}{ Ecopedagogía } & \multicolumn{1}{c}{ Planteamiento } \\
\hline Como parte de la vida cotidiana & $\begin{array}{l}\text { Pretende desarrollar una nueva mirada sobre la educación, una nueva } \\
\text { manera del ser y estar en el mundo, una manera de pensar a partir de la } \\
\text { vida cotidiana, que busca sentido a cada momento y en cada acto. }\end{array}$ \\
Exige se experimente en la práctica, con experiencias educativas dentro \\
y fuera del sistema educativo.
\end{tabular}

Fuente: Zingaretti, 2008.

Con esta breve revisión sobre la ecopedagoia o pedagogía de la Tierra, tratamos de mostrar que la educación debe centrarse en la ética y en la filosofía, debemos preguntarnos cómo debemos ser para aprender y qué necesitamos saber para aprender a enseñar. Este y otros análisis pueden revisarse más a detalle en Gadotti.

Es importante señalar que tenemos la tarea pendiente de incorporar a la sociología de la educación para entender a la educación en su dimensión social, pues tiene un fundamento moral que pretende moldear la conducta del hombre/mujer como ser social y a su vez la de mejorar la sociedad. La sociología de la educación tiene dos objetivos mutuamente complementarios: la constitución histórica de los sistemas 
educativos, de cuya comprensión se desprenden las causas que los originaron y los fines que cumplen; y la forma en que funcionan en las sociedades contemporáneas. De ahí que la sociología de la educación se convierte en un instrumento metodológico que puede ayudarnos a comprender las formas de comportamiento en los procesos de aprendizaje que estamos impulsando (ETAC, 2008).

\section{ÉTICA Y PRINCIPIOS EN LA IMPLEMENTACIÓN DEL HUERTO ESCOLAR}

La implementación del huerto, se basa en la ética y principios de la Permacultura. Para Ian Lillington (2010), la Permacultura implica:

- $\quad$ El cuidado del planeta, considerando las leyes de la naturaleza,

- $\quad$ Tiene un enfoque positivo, orientado hacia las soluciones y puede ser llevado a la práctica, por cada uno de nosotros en nuestras vidas cotidianas.

- $\quad$ Es un estilo de vida. "Hacer Permacultura" significa observar bien lo que consumimos y aceptar el desafío del cambio. Si se acepta este reto, también implica un aprendizaje sobre información técnica y filosófica necesarias para realizar elecciones informadas, proporcionando el apoyo moral para vivir más éticamente.

Los valores éticos de la Permacultura son los siguientes: 1) cuidado de la tierra, es un componente ecológico que reconoce a la Tierra como la base de toda la vida y por tanto se deben generar valores de respeto y responsabilidad para impulsar acciones en el uso y manejo responsable de los recursos; 2) cuidado a las personas, componente social que considera los derechos de todas las personas a decidir sobre su vida, con libertad y responsabilidad en el uso de los recursos básicos, buscando un apoyo entre nosotros mismos para cambiar hacia maneras de vivir mejor, que no nos dañen ni a nosotros ni al plañera, tratando de alcanzar un equilibrio entre las necesidades individuales y colectivas; 3) reparto equitativo, este componente económico reconoce que la Tierra dispone de recursos finitos, por tanto habrá que establecer límites en nuestras formas de consumo y producción; tenemos el compromiso de salvaguardar recursos para las generaciones futuras (Burnett, 2007).

David Holmgren, citado por Hieronimi (2009), propone desde la Permacultura doce principios que sirven como instrumento para lograr una transición productiva de una sociedad industrial de alto consumo energético hacia una cultura sostenible, y desarrollar una visión de adaptación creativa para un mundo, donde los recursos naturales y la energía serán cada vez más escasos (Figura 2).

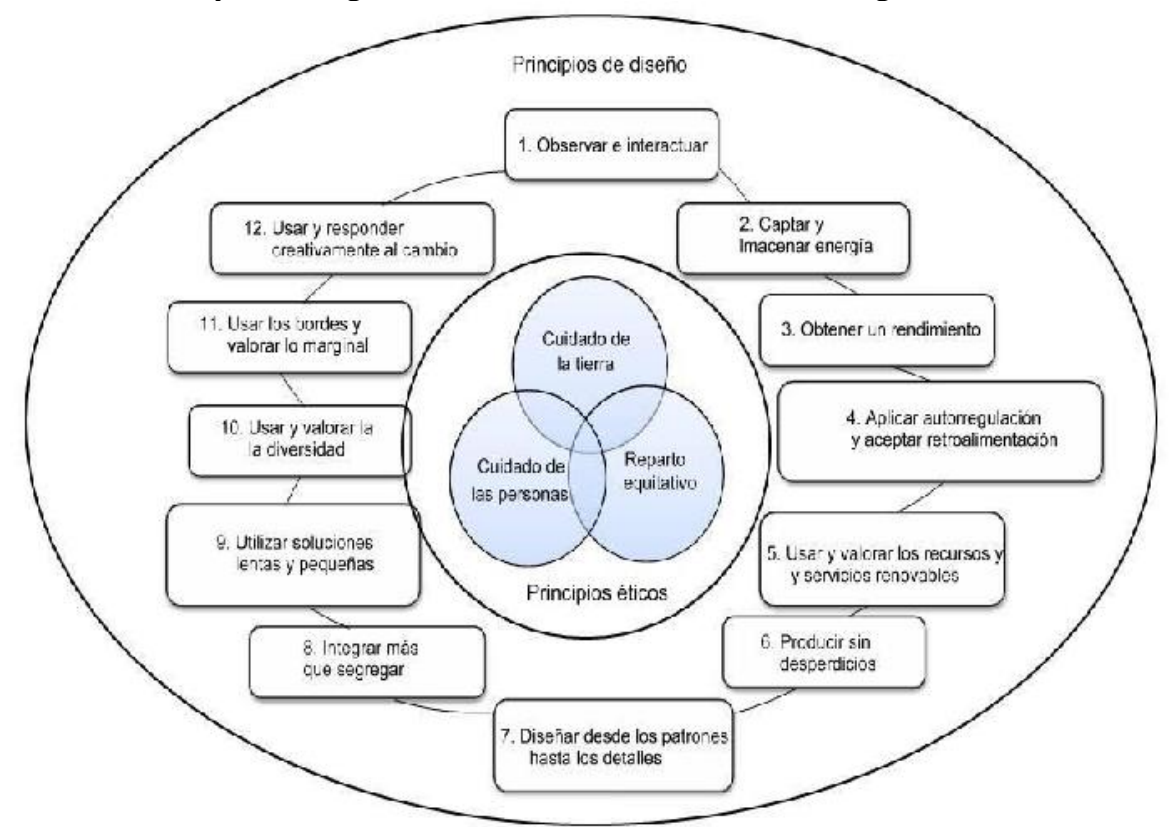

Figura 2. Principios éticos y de diseño de la Permacultura 
Los principios éticos y de diseño son funadamentales tenerlos en cuenta en el establecimiento del huerto, y si bien no se ha logrado consolidar todos los principios de diseño se trabaja para ir cubriendolos.

\section{El sendero que deberíamos conocer: una pedagogía para la vida}

Cuando se implementó el huerto como estrategia educativa, nos planteamos objetivos: educativos (aprender-haciendo), ambientales y nutricionales (Tello et al, 2011). El huerto que se estableció al inicio de este proceso tiene alrededor de $200 \mathrm{~m}^{2}$, sus componentes principales son la fuente de abastecimiento de agua, las camas de producción y él área de composteaje. El método de producción utilizado es el biointensivo propuesto por Jeavons (2002). El huerto ha significado un espacio para poner en práctica principios teóricos y metodológicos que supone la Agroecología, pero al paso de los ya casi cinco años de trabajo, se ha visto la necesidad de ir consolidando un marco de referencia pedagógico que cobije la experiencia; es así que encontramos en la ecopedagogia o pedagogía de la tierra los elementos para la promoción del aprendizaje del sentido de las cosas a partir de la vida cotidiana, pues el huerto supone un espacio que se incorpore como una necesidad y actividad de nuestro trabajo constante (Figura 3). Y también encontramos desde la Permacultura una serie de valores éticos y de diseño, tal y como ya se describieron, que nos van dando pauta para planear las acciones. De esta manera, abrimos nuestra mente para ser receptora de todo el trabajo intelectual que se desarrolla en todas las esferas del conocimiento y que consideramos pueden ser útiles, y sobre todo llevarlas a la práctica para seguir impulsando esta estrategia educativa.

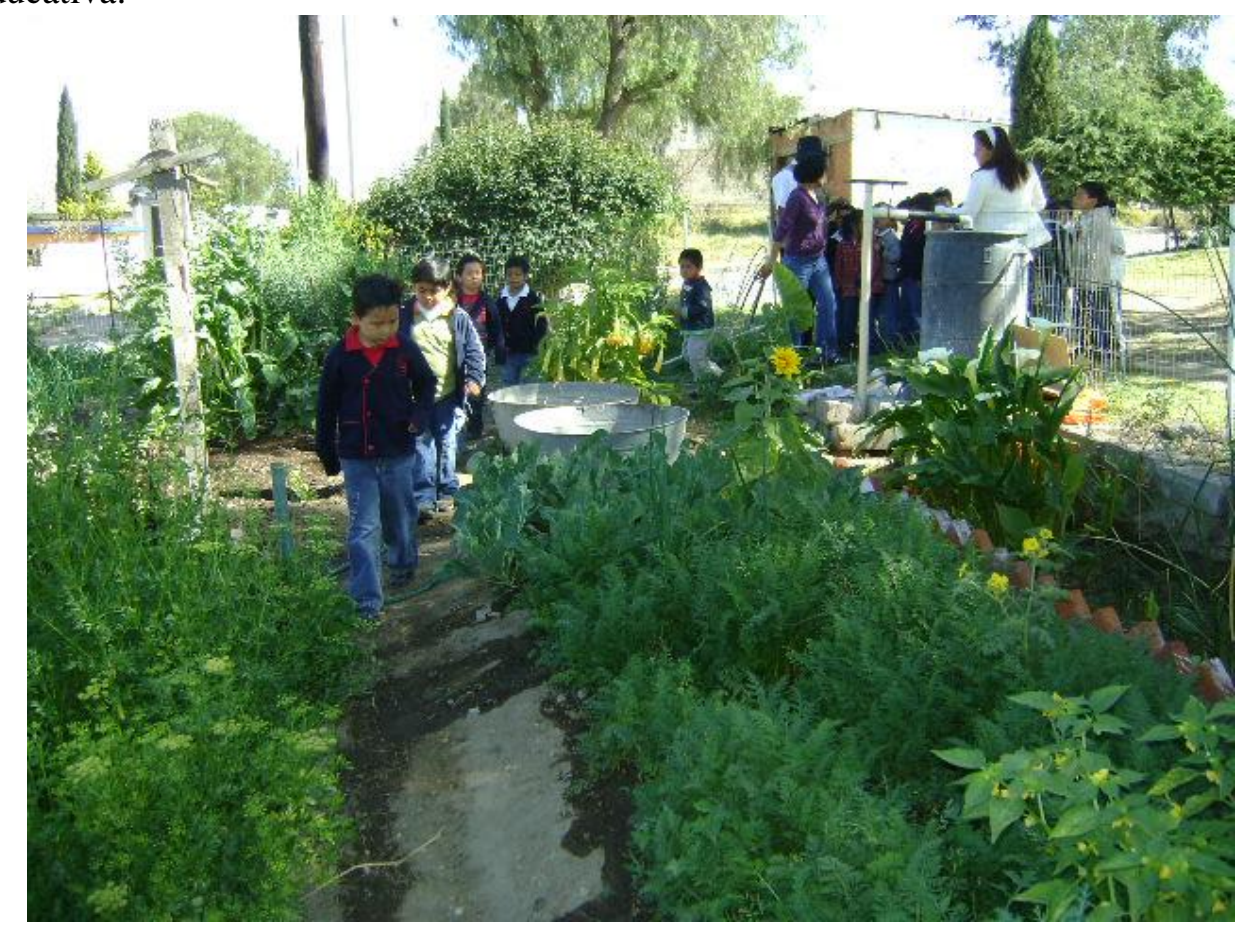

Figura 3. Práctica escolar en el huerto

Lo más rico de esta experiencia ha sido que el huerto que inicialmente se estableció para la escuela de Prescolar "Colegio Ateneo", se ha convertido en un espacio de interacción e intercambio, en donde se ha recibido la visita de estudiantes de medicina tradicional de la Universidad Autónoma Chapingo, alumn@s del Politécnico Nacional de la Facultad de Ingeniería Mecánica, autoridades locales encargadas de los huertos familiares que se impulsan a través DIF; y con quien esta relación de visita ha trascendido más allá de la implementación de otro huerto escolar, es con la Escuela Telesencundaria "Lic. Benito Juarez", ubicada en Tezoyuca, Estado de México, en donde con apoyo de las autoridades escolares y profesoras de los grupos y encargadas de la Comisión del Cuidado del Medio Ambiente, se ha puesto en marcha un programa de reforestación escolar, remozamiento y conservación de áreas verdes, acondicionamiento de 
espacios para la enseñanza como dos foros al aire y establecimiento del huerto escolar; también se ha comenzado con una campaña permanente de separación de residuos sólidos. Y para celebrar fechas conmemorativas sobre el medio ambiente, se convocó a un concurso de dibujo con el tema "la importancia de cuidar el agua”. En síntesis podemos decir que no solo se están impulsando acciones vistas desde la perspectiva productiva, sino que también se ha empezado a incursionar en otros temas relacionados básicamente con una educación ambiental.

Con estas actividades, 1@s alumn@s empiezan a experimentar, a construir con sus compañer@s y a generar experiencias vividas utilizando sus propios conocimientos y los adquiridos en estos espacios. Se quiere que el aprendizaje se pueda dar en la acción.

\section{CONCLUSIONES}

La implementación del huerto, permite generar una experiencia propia, reconociendo las relaciones entre la humanidad y la naturaleza, ya que sin estas relaciones difícilmente encontraríamos el sentido de la vida. El huerto significa la construcción de una estrategia educativa que puede difundirse por sí misma, dada la necesidad que tenemos de buscar otras opciones de enseñar y aprender y sobre todo que es una forma tangible de expresar nuestro talento.

Si bien nuestra experiencia tiene un impacto local, hemos logrado impulsar una educación que nos hace sentir que somos miembros de la Tierra. Sin embargo, acorde con los planteamientos desde la ecopedagogia, debemos trabajar para difundir la Carta de la Tierra, como un instrumento de fuerza para luchar sobre la base de un consenso global.

Estamos trascendiendo a otros escenarios de enseñanza que sobrepasan el ámbito productivo a acciones relacionadas con la educación ambiental, sin embargo, con las actividades realizadas volvemos a caer en la lógica de una educación fundada en la competitividad, donde recurrimos a un sistema de premios para incentivar la participación.

Sin duda, nos hace falta fortalecer valores de confianza y negociar entre todos para impulsar un proceso de cambio.

\section{LITERATURA CITADA}

Alcorta, D. 2000. Lecciones de filosofía, México, Ed. EDESA

Antunez y Gadotti. 2000. La ecopedagogía como la pedagogía indicada para el proceso de la Carta de la Tierra. Ensayo temático que se refiere al Principio 14 sobre incorporar los valores de la Carta de la Tierra a la educación. La Carta de la Tierra en Acción. IV Democracia, no violencia y paz. Brasil pp140-143.

Burnett, Graham. 2007. Permacultura. Una guía para principiantes. Academia de PC, Eco habitat España.

Gadotti, M. 2002. Pedagogía de la Tierra. Siglo XXI editores, S.A. de C.V.

Gutiérrez F. y Prado C. 2004. Ecopedagogia y ciudadanaia planetaria.

Hieronimi, H. 2009. Curso Fundamentos de la Permacultura. 15-20 Agosto, 2009. Eronguaricuaro, Mich., México.

Jeavons J. 2002. Cultivo biointensivo de alimentos: más alimentos en menos espacio. Ecology action of the MidPeninsula. Traducido por Wille Castillejos, edición de Juan Manuel Martínez y Adriana Guzmán. Estados Unidos de Norteamérica.

Lillington, I. 2010. La Permacultura según Ian Lillington. Escrito por Administrator 13 de Noviembre de 2002 Actualizado 12 de Octubre de 2010. http://www.permacultura-es.org/iqus-la-permacultura-mainmenu89/1481-la-permacultura-segan-lillington.pdf

Mallart, N., J. 2010. Es la hora de la ecopedagogía. La década de la educación para un futuro sustentable.

ETAC. 2008. Material Didáctico, Maestría en Ciencias de la Educación: Filosofía y sociología en la educación, México.

Tello, G., E, y Rodríguez, H., B., Aguilar C., S. 2011. Huerto agroecológico "un pasito en grande”: estrategia educativa por un futuro sostenible y una vida saludable. En Pérez O., Ma. A (Coord). Horticultura, 
Experiencias productivas con fines educativos y de capacitación. Pp51-87. Colegio de Posgraduados en Ciencias Agrícolas, Montecillos, Estado de México.

Zingaretti, H. 2008. La ecopedagogía y la formación en los niños. X Congreso Nacional y II Congreso Internacional. "Repensar la niñez para el siglo XXI", Mendoza.

\section{Benito Rodríguez-Haros}

Profesor de la Universidad de Guanajuato Campus Celaya-Salvatierra. Privada de Arteaga S/N Salvatierra Guanajuato, CP 38900.

\section{Enriqueta-Tello-García}

Investigadora Titular del Programa de Estudios para el Desarrollo Rural, Colegio de Posgraduados. Carretera Federal México -Texcoco Km 36.5 , CP 56230.

\section{Salvador-Aguilar-Californias}

Director General del Colegio Ateneo, Educación Prescolar. Calle 21 de Marzo Col. San Felipe Tezoyuca, Estado de México. CP 56000. 